\title{
VOICE AND GESTURE BASED HOME AUTOMATION SYSTEM
}

\author{
Pradeep M, Ragul K and Varalakshmi K
}

\begin{abstract}
It is now common to control home appliances and electronic gadgets through an Infrared remote control. These tasks can also be done more easily. The primary motive of proposing a new system of hand gesture control is to eliminate the need for the elderly/disabled people to use a physical remote but rather use simple gestures. Gesture means a movement of part of body. Gesture Recognition is the technology that is used to identify physical actions. It recognizes hand, arms, head or any part of the body. So the goal is to provide a human interface to the computer. The devices can be controlled not only by using gestures but also by using voice commands as well. Smart assistant such as Google assistant can be used for this purpose.
\end{abstract}

Keywords - IoT, Arduino, Flex, Google Assistant, Gesture and Voice commands.

Reference to this paper should be made as follows:

Pradeep M, Ragul K and Varalakshmi K, "Voice and Gesture Based Home Automation System” Int. J. Electronics Engineering and Applications, Vol. 9, No. 1, pp. 08-18, doi 10.30696/IJEEA.IX.I.2021.08-18. 


\section{[I] INTRODUCTION}

We live in an exciting generation where commonly used everyday appliances i.e. "things" are becoming smart! "Things" that have sensors can communicate to other "things" and can provide control to more "things". The Internet of Things (IoT) is upon us in a huge way and people are rapidly inventing new gadgets that enhance our lives. Smart Building not only refers to reduce human efforts but also energy efficient and time saving. In this paper, everyday appliances are connected to sensors and sensors give status of appliances to the web. Controlling home appliances and electronics gadgets through an Infrared remote control is now in general. But the same controlling tasks can be done more easily. Primary motive of proposing the new system of hand gesture remote control is to remove the need to look in to the hand held remote and to search for a specific key for specific function. Gesture means a movement of part of body. Gesture Recognition is the technology that recognizes the movement of body. It recognizes hand, arms, head or any part of the body. So, the goal of Gesture Recognition is to provide interface of human body with computer via mathematical algorithm. This paper presents a novel system to control home appliances through hand gesture as a remote-control device. In this paper, not only by gestures the devices are controlled by using voice with the help of digital assistant like Google Voice Assistant [1-4].

\section{[2] LITERATURE REVIEW AND PREVIOUS WORK}

The construction and conversion of ordinary homes into "smart homes" has seen a tremendous rise in recent years. This can be ascribed to technologies such as the Internet of Things, sensors, smart phones, smart appliances, cloud computing, and digital assistants such as Amazon Alexa, Google Home, Google Assistant, Apple Siri, and Microsoft Cortana [5]. At the outset, smart homes were built to enhance the quality of life for ordinary non-disabled persons. Impressively, we have seen smart home residents reaping the benefits of security, energy saving, and the ability to control their lighting, HVAC (heating, ventilation, and air conditioning), door locks, and coffee makers while they are in their space of comfort, for example in bed or sitting on a couch. [6-8] However, most smart home devices are not designed with people with disabilities and limited range of movement in mind. Of course, being able to control home devices using smart technology could be a tremendous benefit to people with physical disabilities and the older persons. This paper presents a system that uses smart plugs, smart cameras, smart power strips and a digital assistant such as Amazon Alexa, Google Home, Google Assistant, Apple Siri, or Microsoft Cortana to capture voice commands, from a person with physical disabilities, spoken in a much more natural way to control ordinary home electrical appliances in order to turn them on or off, with minimal exertion [9].

The current populace of the elderly is apparently abandoned by the younger generations due to their individual circumstances. To heighten the vitality and strengthen the fitness of elders, assisting a home care system can be an admittance that provides comprehensive nursing and monitoring them in the regular interim [10-11]. To deliver an interactive service supervision platform to the elders a smart environment of various sensors is clubbed together to establish an intuitive platform that can control the home appliances and gadgets within the living space of elders. The proposed system used voice and gesture (MPU6050 accelerometer) to control the home appliances like turning on/off the light, closing/opening of curtains, TV, and fan or AC within the living spaces. The system also monitors the real-time activity like heart rate and body temperature for the elderly citizens. In the case of emergency, for instance, anomalous behaviors like heart stroke occurs, the 
proposed system set-up triggers an alarm and the emergency bulb will be strikes "on" to alert their kin. This smart environment can set the temperature and help control the living parameters based on the users' comfort and their health conditions. The whole design is to provide modest support systems for the elder to live healthily and safely in an independent living environment [12].

This paper discusses a remote-control system of home electrical appliances using speech recognition. It is very convenient system for not only visual-impaired people but also elderly people to control household appliances based on the speech commands. The goal of our system is that the many kinds of household appliances such as television, video recorder and air conditioner are controlled based on the speech commands. The feature of our remote-control system is that the system has human friendly interface just as persons communicate with each other. When the system did not recognize a person's speech command, the system asks once more for a speech command to repeat what a person said. The experimental results for our system have clarified its effectiveness [13-14].

All finished world, deaf and dumb individuals confront battle in communicating their emotions to other individuals. There are different difficulties experienced by discourse and hearing weakened individuals at open places in conveying everything that needs to be conveyed to ordinary individuals. The answer for this issue is resolved in this paper, by the utilization of the Indian gesturebased communication images which are bland to all deaf and dumb individuals in India. The motions outlined by the Indian gesture-based communication images will be vanquished with the help of the flex sensors and accelerometer. The developments included amid signal portrayal are turn, point tilt, and heading changes. The flex sensor is consolidatedover fingers of two and accelerometer sensor over the wrist separately to obtain their progression, these sensors are fitted over the information glove. The one hand fingers are utilized to control home apparatuses like light fan and entryway while the other hand finger is utilized to send message.

\section{[3]OUR SYSTEM'S ARCHITECTURE AND IMPLEMENTATION}

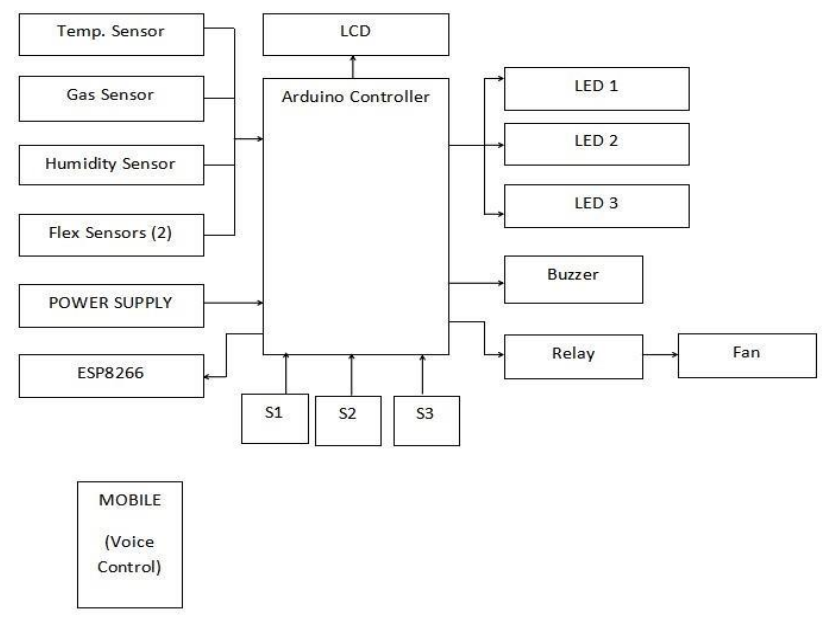

Fig. 1. System Architecture

Arduino Uno is the brain of our project,I.e. the main control unit. This unit has several other hardware components integrated with it namely flex sensor, gas sensor, temperature and humidity sensor, LCD display, Wi-Fi module etc.... Power board is used in order to provide power supply to the hardware components. DC adapter is used to give the power supply to board via power sockets. 
The device is controlled by Voice commands and Gesture commands. The voice commands are given through Android mobile which uses an Android Application. The android application is built with the function of using the Google voice assistant (Digital Assistant). Voice commands like "Light 1 ON" and "Light 1 OFF" represents the ON|OFF state of the LED light present. Similarly, LED lights 2 and 3 also have the same voice commands "Light 2 ON" \& "Light 2 OFF" / "Light 3 ON" \& "Light 3 OFF". Based on these commands we can ON and OFF the LED lights as per our wish.

On the other hand, we have Gesture based commands, which controls the electrical appliances using the flex sensor connected with the main micro-controller. The flex sensor can take two inputs, if the sensor is straight, it is considered to an input and if the sensor is bent, it is considered as another input. We use two flex sensors in our project. We can name it flex 1 and flex 2 . If flex 1 is bent, the light 1 will be turned ON and if it is set as straight the light will turn OFF. Similarly, if flex2 is bent light 2 will be turned On and if it is set as straight the light will turn OFF. When both the flex 1 and flex 2 are bent together, light 3 will turn $\mathrm{ON}$ and when both are straight light 3 will be turned OFF.

Switches are also used for the control of the lights and also it is controlled from the IoT. Temperature sensor, Gas sensor and Humidity sensor are used for the monitoring of temperature, gas and humidity and it is displayed in the MQTT through ESP8266. When the temperature gets increased and based on the threshold, the Buzzer will get alerted. Similarly, when the temperature value is above 40, the Fan will also be turned ON and OFF.

The values from these sensors are displayed on the LCD display so that the user gets to know what happened. We introduce a simple smart home system used to control the existing non-smart electrical appliances. The whole system is based on a main control unit and input/output unit. An Arduino microcontroller is the core of the whole system. It can be used to turn on or off the lights, power switch, door lock, various electric appliance switches.

The main unit also have the IoT Module, the owner can program to the unit through the internet. Flex sensors are used to provide gesture inputs to the controllers. These inputs are processed by the controller and control the devices. Digital assistant, namely google assistant is used to provide voice inputs.

\section{[3] OUTPUT SCREENSHOTS}

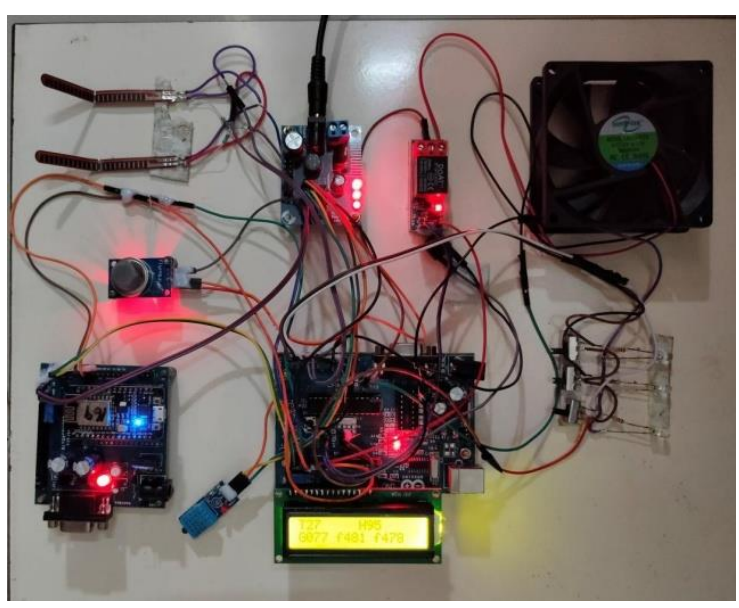

Fig. 2. LCD Display 


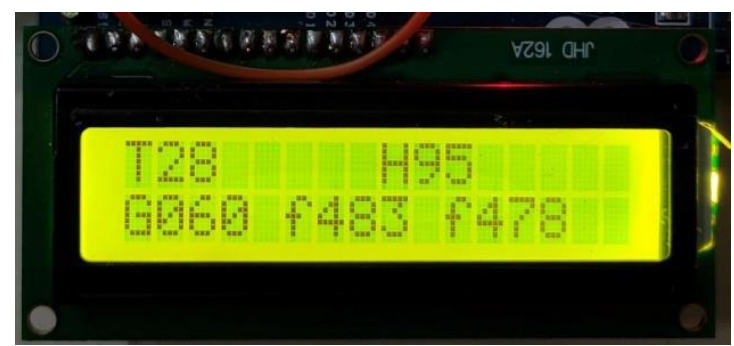

Fig. 3. System Default State

The Smart Home System is in a default state where all the sensors and modules are initialized. The values of various sensors are displayed in the LCD. The Gas concentration value $\mathrm{G}$ is below the threshold value of 100 , so the fan is in Off state. When it exceeds the threshold value, the fan automatically turns $\mathrm{ON}$.

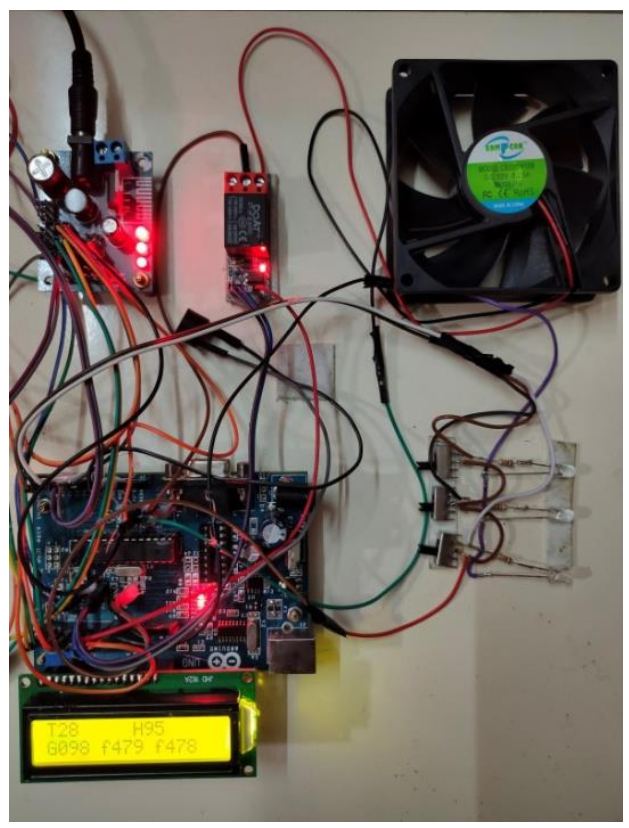

Fig. 4. Gas Concentration < 100, Fan OFF

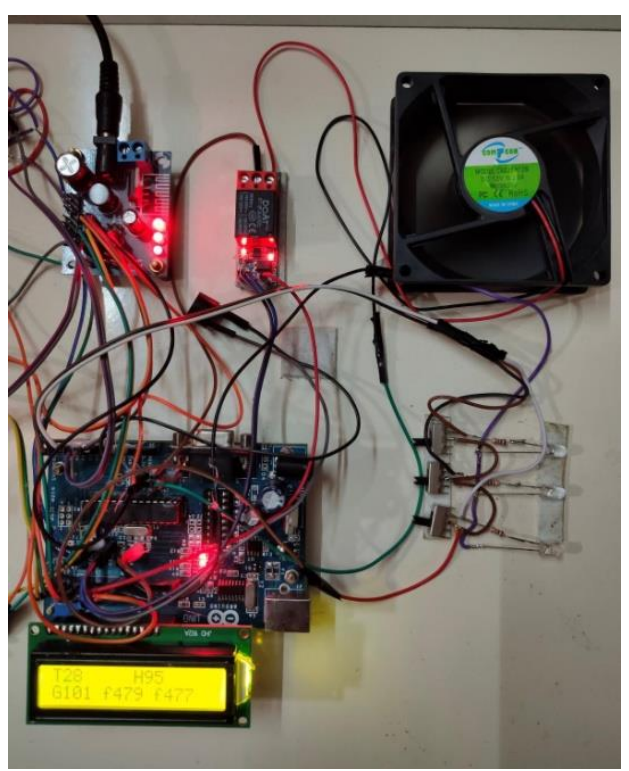

Fig. 5. Gas concentration $>100$, Fan $\mathrm{ON}$ 
The Smart Home System is connected with an Android Smart phone using Wi-Fi module (ESP8266). The Wi-Fi module is connected to the IoT Board V2 which has default SSID and Password IOT data \& 12345678 respectively. This default SSID and Password should be used to create a hotspot connection on the android smart phone.

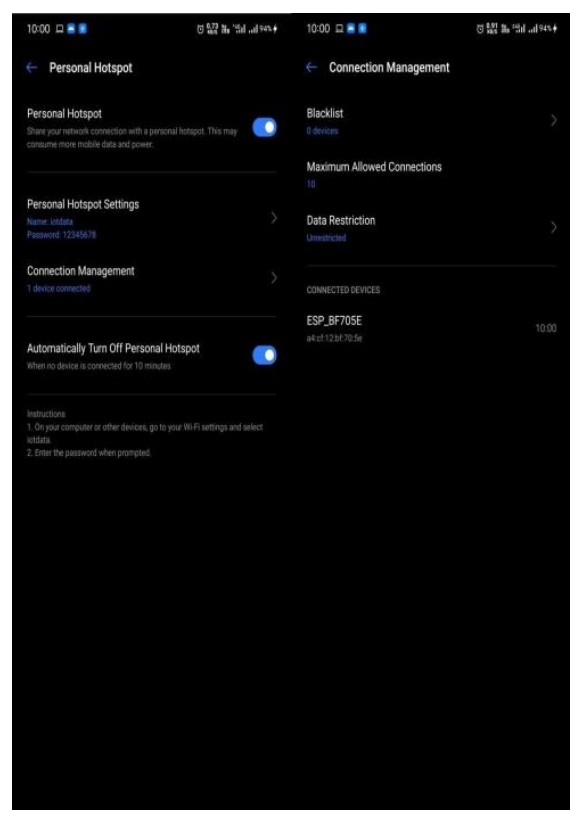

Fig. 6. Connecting Mobile Device

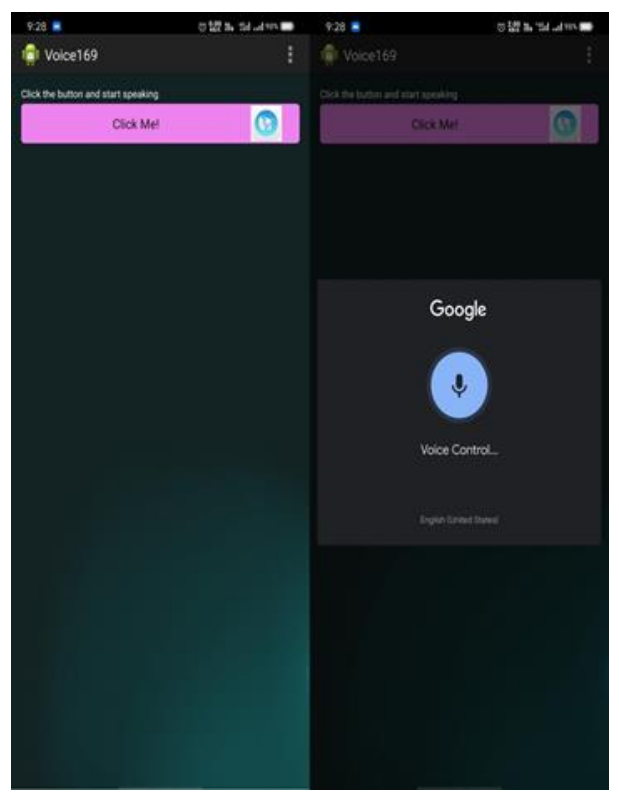

Fig. 7. Android Application UI

The Android Application we developed using Android Studio has a simple design. It contains a single button "Click Me!" that is used to invoke the Google Assistant. On clicking the aforementioned button, a pop-up appears asking for the user's voice input.

After clicking the button, the user gives the voice command "Light one ON", which is interpreted by the Google Assistant and the input is sent to the Arduino micro-controller using the Wi-Fi module. 
Pradeep M et al.

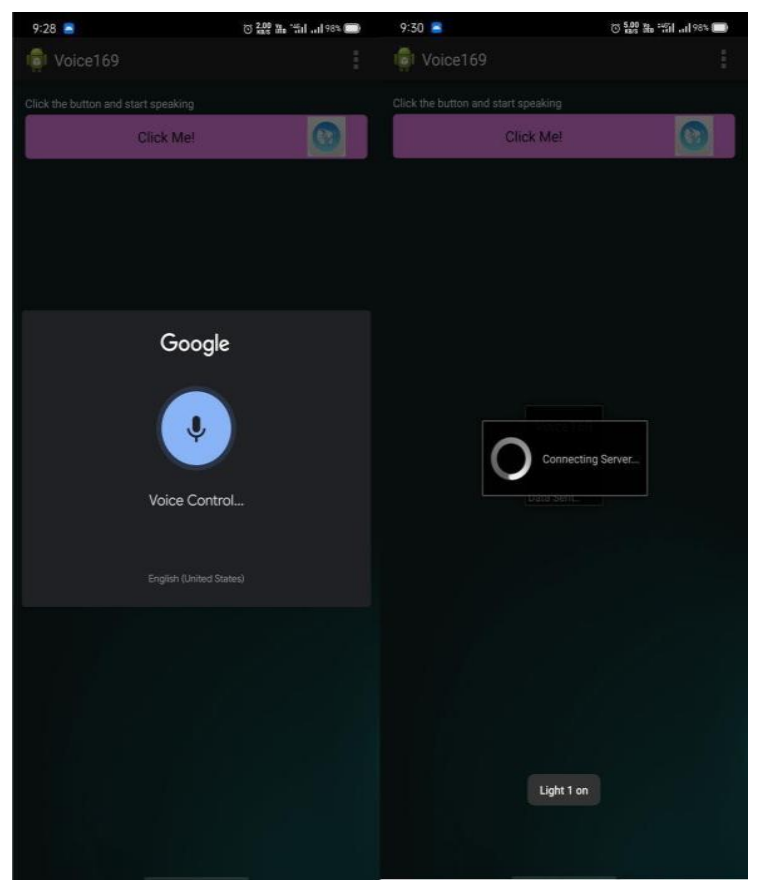

Fig. 8. Voice Input - Light 1 ON

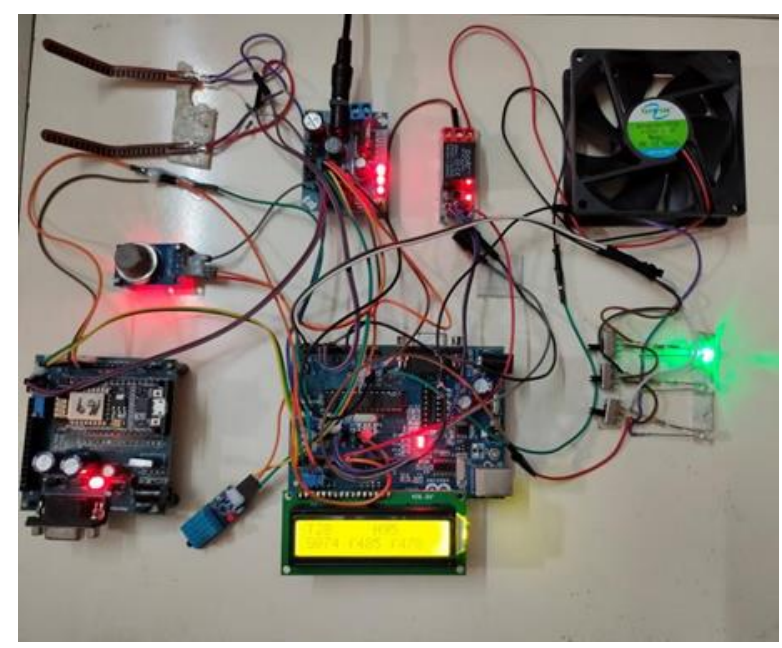

Fig. 9. Light 1 ON

After clicking the button, the user gives the voice command "Light two ON", which is interpreted by the Google Assistant and the input is sent to the Arduino micro-controller using the Wi-Fi module. 


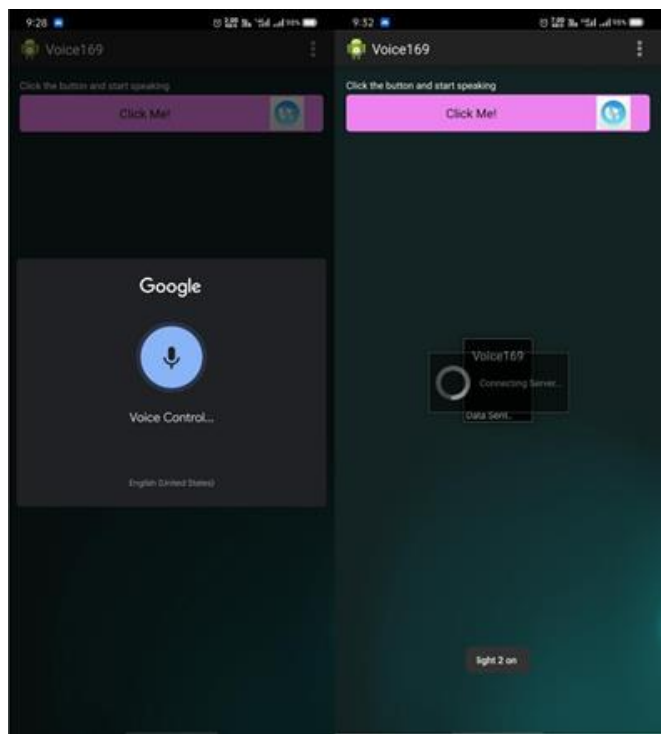

Fig. 10. Voice Input - Light 2 ON

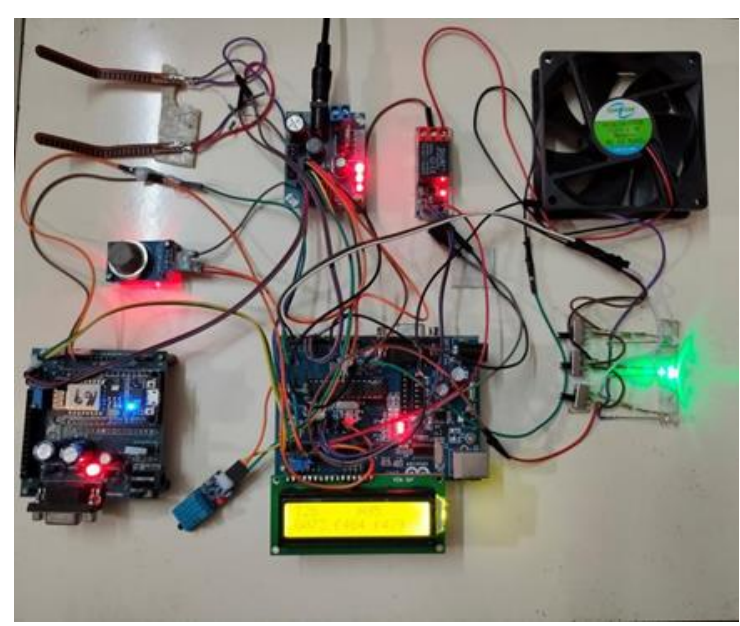

Fig. 11. Light ON

After clicking the button, the user gives the voice command "Light three ON", which is interpreted by the Google Assistant and the input is sent to the Arduino micro-controller using the Wi-Fi module.

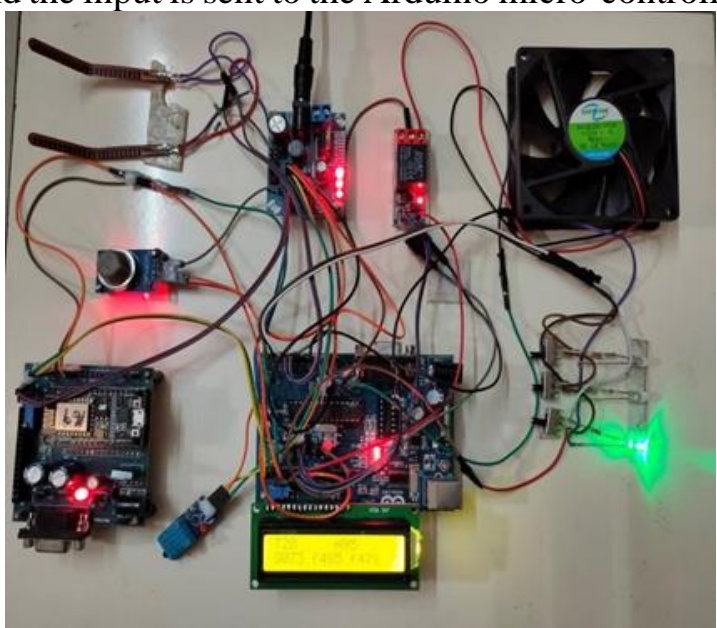

Fig. 10. Light 3 ON 
Pradeep M et al.

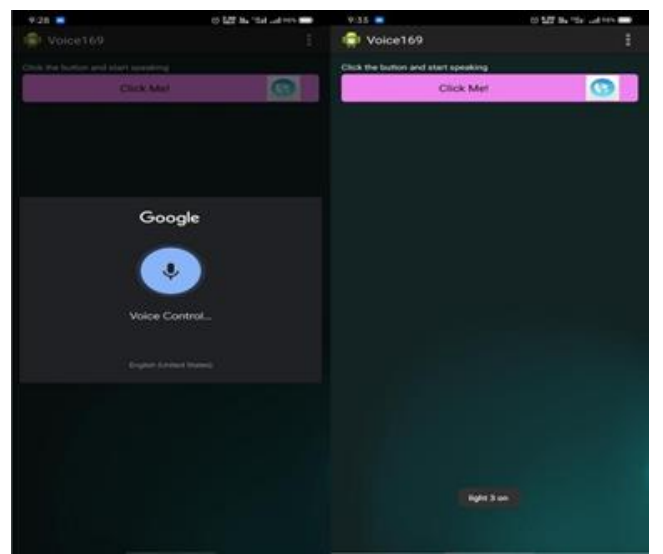

Fig. 11. Voice input - Light $3 \mathrm{ON}$

The flex sensor 1 is bent beyond the threshold limit, this sends a signal to the Arduino microcontroller to switch ON light 1.

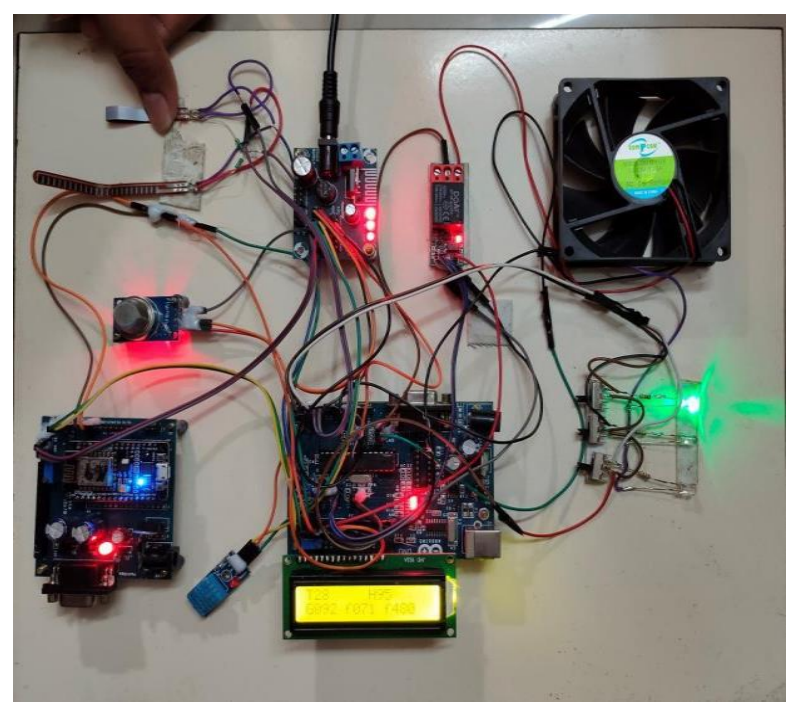

Fig. 12. Light 1 switched ON using Flex sensor

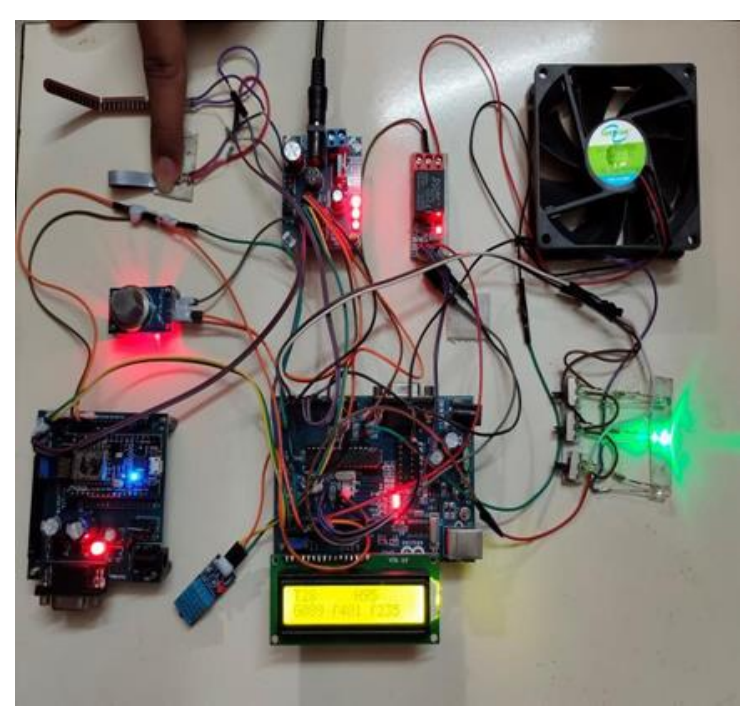

Fig. 13. Light 2 switched ON using Flex sensor 
The flex sensor 2 is bent beyond the threshold limit, this sends a signal to the Arduino microcontroller to switch ON light 2.

The flex sensors 1 and 2 are both bent beyond the threshold limit, this sends a signal to the Arduino micro-controller to switch ON light 3.

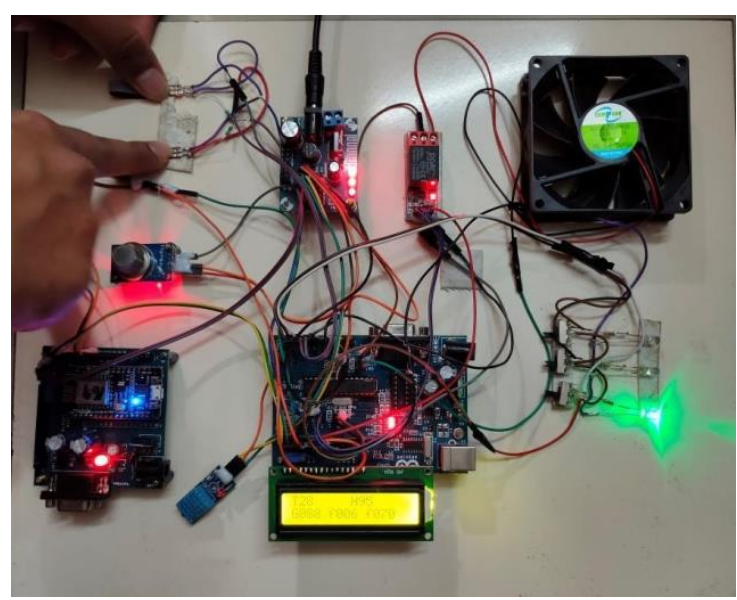

Fig. 14. Light 3 switched ON using Flex sensor

\section{[4] CONCLUSION}

This paper describes and provides a prototype that addresses many prevalent issues in the current system in order to achieve an efficient and cost-effective Smart home system that can be used by differently abled people as well. We are using Google Assistant as Digital Assistant, but we can also add other existing digital assistants like Amazon Alexa, Apples Siri etc... So that we can improve the input giving flexibility. We can also add Accelerometer Sensor, so that we can increase the range of gestures which improves the input providing ways. Adding additional Flex sensors leads to multiple input recognition, and thus various actions can be performed by an individual. 


\section{REFERENCES}

[1] H. Basanta, Y. Huang, and T. Lee, “Assistive Design for Elderly Living Ambient using Voice and Gesture Recognition System”, International Conference on Systems, Man, and Cybernetics (SMC), 2017.

[2] Munirathnam S, Harika P, “Automated Gesture Control System Using IOT”, International Journal of Engineering Trends and Applications (IJETA), 2018.

[3] Noriyuki Kawarazaki and Tadashi Yoshidome, "Remote Control System of Home Electrical Appliances Using Speech Recognition" 8th IEEE International Conference on Automation Science and Engineering, 2012.

[4] Progress Mtshali and Freedom Khubisa "A Smart Home Appliance Control System for Physically Disabled People", Conference on Information Communications Technology and Society (ICTAS), 2019.

[5] Sakshi Bajpai and D. Radha, "Smart Phone as a Controlling Device for Smart Home using Speech Recognition", International Conference on Communication and Signal Processing, 2019.

[6] Siddharth Wadhwani, Uday Singh, Prakarsh Singh, Shraddha Dwivedi, "Smart Home Automation and Security System using Arduino and IOT", International Research Journal of Engineering and Technology (IRJET), 2018.

[7] J. Waleed, A. M. abduldaim, T. M. Hasan, and Q. S. Mohaisin, "Smart Home as a New Trend, a Simplicity Led to Revolution”, 1st International Scientific Conference of Engineering Sciences, 2018

[8] Yash Mittal, Paridhi Toshniwal, Sonal Sharma, Deepika Singhal, Ruchi Gupta V. K. Mittal, "A VoiceControlledMulti-Functional Smart Home Automation System", International Conference on Automation Science and Engineering, 2015.

[9] Anoop Joyti Sahoo, and Rajesh Kumar Tiwari "A Novel Approach for Hiding Secret data in Program Files” International Journal of Information and Computer Security. Volume 8 Issue 1, March 2016,

[10] Abu Salim, Sachin Tripathi and Rajesh Kumar Tiwari "A secure and timestamp-based communication scheme for cloud environment" Published in International Journal of Electronic Security and Digital Forensics, Volume 6, Issue 4, 319-332.

[11] Rajesh Kumar Tiwari and G. Sahoo, "A Novel Watermark Scheme for Secure Relational Databases" Information Security Journal: A Global Perspective, Volume 22, Issue 3, July 2013.

[12] Chen, Alvin I., Max L. Balter, Timothy J. Maguire, and Martin L. Yarmush. "Deep learning robotic guidance for autonomous vascular access." Nature Machine Intelligence 2, no. 2 (2020): 104-115.

[13] Zillner, Jakob, Erick Mendez, and Daniel Wagner. "Augmented reality remote collaboration with dense reconstruction." In 2018 IEEE International Symposium on Mixed and Augmented Reality Adjunct (ISMAR-Adjunct), pp. 38-39. IEEE, 2018.

[14] Chen, Po-Hsuan Cameron, Krishna Gadepalli, Robert MacDonald, Yun Liu, Shiro Kadowaki, Kunal Nagpal, Timo Kohlberger et al. "An augmented reality microscope with real-time artificial intelligence integration for cancer diagnosis." Nature medicine 25, no. 9 (2019): 1453-1457 